\title{
EHMTI-0293. Comparison between celecoxib and prednisolone in bridge stage therapy following medication overuse headache: double blind randomized clinical trial
}

\author{
M Togha ${ }^{1 *}$, SMH Paknejad', E Taghvaei ${ }^{1}$, T Ramim $^{1}$, S Razeghi jahromi ${ }^{2}$ \\ From 4th European Headache and Migraine Trust International Congress: EHMTIC 2014 \\ Copenhagen, Denmark. 18-21 September 2014
}

\section{Introduction}

Many patients with chronic or frequent headaches overuse the analgestic or anti-migraine drugs. Medication overuse headache $(\mathrm{MOH})$ is a common severe headache. The basis of treatment of $\mathrm{MOH}$ is discontinuation of the offending drugs. However, cessation of drug consumption could lead to severe disabling headache. Corticosteroids are the most popular drugs for bridge phase of treatment. However corticosteroids might cause different side effects. Celecoxib is a Cox 2 inhibitor drug with analgesic and antiinflammatory properties, assumed to be a good alternative for steroids.

\section{Aims}

We conducted this study to compare celecoxib with prednisolone for bridge stage therapy in $\mathrm{MOH}$.

\section{Methods}

103 patients (18-65 years) with at least 15 days of headache per month were enrolled. They assigned into two groups: celecoxib (53 cases) and prednisolone (51 cases). Prednisone was administered as daily doses of $75,50,30$, 25 , and $10 \mathrm{mg}$ in 3 days interval. Celecoxib was administered for 15 days according to the following schedule: 100 $\mathrm{mg}$ three times per day (5 days), twice a day ( 5 days) and once a day (5 days). The severity and duration of headache, as well as side effects were assessed.

\section{Results}

No significant differences were observed in the decrement of the severity and duration $(\mathrm{p}=0.114$ and 0.149 respectively) of headache between two groups. Side effects were reported in $42 \%$ and $18.9 \%$ of prednisolone and celcoxibe groups respectively (Relative Risk $=2.2$, $\mathrm{p}=0.011$ ).

\section{Conclusion}

Celecoxib has similar efficacy and fewer side compared to prednisolone during withdrawal therapy.

No conflict of interest.

\section{Authors' details}

${ }^{1}$ Iranian Center of Neurological Research-Neuroscience Institute, Tehran University of Medical Sciences, Tehran, Iran. ${ }^{2}$ Multiple Sclerosis Research Center-Neuroscience Institute, Tehran University of Medical Sciences, Tehran, Iran.

Published: 18 September 2014

\section{doi:10.1186/1129-2377-15-S1-C54}

Cite this article as: Togha et al:: EHMTI-0293. Comparison between celecoxib and prednisolone in bridge stage therapy following medication overuse headache: double blind randomized clinical trial. The Journal of Headache and Pain 2014 15(Suppl 1):C54. 